PROCEEDINGS OF THE

AMERICAN MATHEMATICAL SOCIETY

Volume 137, Number 12, December 2009, Pages 4177-4185

S 0002-9939(09)09983-3

Article electronically published on June 25, 2009

\title{
A NEW INTERPOLATION FORMULA FOR THE TITCHMARSH-WEYL $m$-FUNCTION
}

\author{
ALEXEI RYBKIN AND VU KIM TUAN \\ (Communicated by Nigel J. Kalton)
}

\begin{abstract}
For the Titchmarsh-Weyl $m$-function of the half-line Schrödinger operator with Dirichlet boundary conditions we put forward a new interpolation formula which allows one to reconstruct the $m$-function from its values on a certain infinite set of points for a broad class of potentials.
\end{abstract}

\section{INTRODUCTION}

The Titchmarsh-Weyl $m$-function is a central object in the spectral theory of ordinary differential operators due to its connection to spectral measures. Its actual numerical computation however is problematic, as one needs to solve a SturmLiouville problem on an infinite interval for every value of the spectral parameter from $\mathbb{C}^{+}:=\{\lambda: \operatorname{Im} \lambda>0\}$. This presents a real computational challenge.

On the other hand, as an analytic function, the Titchmarsh-Weyl $m$-function is uniquely determined by its values on a countable set $\left\{\lambda_{k}\right\}$ with accumulation point in the domain of analyticity of $m(\lambda)$. To the best of our knowledge, the first explicit formula for interpolating the Titchmarsh-Weyl $m$-function from its values on a countable set of complex points with no accumulation point appeared in the paper of Boumenir-Tuan [3] under quite restrictive conditions on the potential. In the current paper we obtain a simpler interpolation formula for $m(\lambda)$ under very mild conditions on the potential. The main advantage of the interpolation formula presented here over the one given in 3 is that it requires us to sample the Titchmarsh-Weyl $m$-function only on the negative half-line. The crucial ingredient is Simon's representation of the Titchmarsh-Weyl $m$-function as a specially interpreted Laplace transform of the so-called $A$-amplitude 8 . Note that in the context of short-range potentials a similar representation was obtained in the 1980's by Ramm [7, who called the $A$-amplitude the " $I$-function".

However, for our purposes Simon's representation is suitable only if it is absolutely convergent. Gesztesy-Simon [5] have proved the absolute convergence for

Received by the editors October 28, 2008, and, in revised form, April 2, 2009.

2000 Mathematics Subject Classification. Primary 47E05, 65D05.

Key words and phrases. Titchmarsh-Weyl $m$-function, interpolation, $A$-amplitude, Hardy space.

This research was supported in part by the U.S. National Science Foundation under grant DMS 070747 . 
$L^{1}$ and $L^{\infty}$ potentials. In [2] Avdonin-Mikhaylov-Rybkin extended the absolute convergence of the Laplace transform representation of the Titchmarsh-Weyl $\mathrm{m}$ function to any locally $L^{1}$ and globally $L^{\infty}$ potential (a precise definition will be given later). Combined with a general statement regarding the interpolation of the Laplace transform similar to the one in [3] for the Fourier transform, this produces our main result. As a by-product, one obtains a rational approximation of the Titchmarsh-Weyl $m$-function.

We note that while our interpolation problem resembles the Nevanlinna-Pick interpolation problem (see, e.g., [1), there are some fundamental differences. The main one is that our approach is essentially real and based upon Legendre polynomials, whereas the Nevanlinna-Pick interpolation problem is based on complexanalytic techniques of Hardy space theory (see Remark 7 for more discussion).

The paper is organized as follows. In Section 2 we give the Laplace transform type representation of the Titchmarsh-Weyl $m$-function suitable for our interpolation. In Section 3 we prove an interpolation formula (Theorem 4) for the general Laplace transform. Finally, Section 4 combines the results of the previous sections to state and prove our main interpolation result.

\section{The LAPLACE TRANSFORM REPRESENTATION OF THE TITCHMARSH-WEYL $m$-FUNCTION}

Consider the Schrödinger operator

$$
H=-d^{2} / d x^{2}+q(x)
$$

on $L^{2}(0, \infty)$ with a real-valued locally integrable potential $q$. We assume that (1) is in the limit point case at $\infty$, that is, for each $\lambda \in \mathbb{C}^{+}:=\{\lambda \in \mathbb{C}: \operatorname{Im} \lambda>0\}$ the equation

$$
-u^{\prime \prime}+q(x) u=\lambda u
$$

has a unique, up to a multiplicative constant, solution $u_{+}$which is in $L^{2}$ at $\infty$ :

$$
\int_{\mathbb{R}_{+}}\left|u_{+}(x, \lambda)\right|^{2} d x<\infty, \quad \lambda \in \mathbb{C}^{+} .
$$

Such a solution $u_{+}$is called a Weyl solution and its existence for a very broad class of real potentials $q$ is the central focus of Titchmarsh-Weyl theory.

The (principal or Dirichlet) Titchmarsh-Weyl $m$-function, $m(\lambda)$, is defined for $\lambda \in \mathbb{C}^{+}$as

$$
m(\lambda):=\frac{u_{+}^{\prime}(0, \lambda)}{u_{+}(0, \lambda)}
$$

The following theorem from [2] will play a crucial role in our considerations.

Theorem 1. If $q$ is real and in $l^{\infty}\left(L^{1}\right)$, i.e.,

$$
\|q\|:=\sup _{x \geq 0} \int_{x}^{x+1}|q(x)| d x<\infty,
$$


then

(6) $m\left(-\kappa^{2}\right)=-\kappa-\int_{0}^{\infty} A(x) e^{-2 \kappa x} d x, \quad \operatorname{Re} \kappa>\gamma:=\max \{\sqrt{2\|q\|}, e\|q\|\}$,

where the integral in (6) converges absolutely. The function $A(x)$ (the A-amplitude) is given by

$$
A(x):=A(x, x),
$$

where $A(x, y)$ solves the linear Volterra-type integral equation

$$
A(x, y)=q(x)-\int_{0}^{y}\left(\int_{v}^{x} A(u, v) d u\right) q(x-v) d v, \quad x, y \geq 0 .
$$

Remark 2. Under condition (5) the spectrum $\sigma(H)$ of $H=-d^{2} / d x^{2}+q(x), u(0)=$ 0 , is bounded from below and

$$
E_{0}:=\inf \sigma(H)>-(\max \{\sqrt{2\|q\|}, e\|q\|\})^{2} .
$$

It is well-known that the Titchmarsh-Weyl $m$-function is then analytic and real valued for $\lambda<E_{0}$ and can therefore be analytically continued into the lower half plane $\mathbb{C}_{-}$by the symmetry principle. In fact, it is easier and more natural in our setting to deal with both half planes at once.

\section{Interpolation OF THE LAPLACE TRANSFORM}

It is well-known that a function $F$ belongs to the Hardy space $H^{2}\left(\mathbb{C}^{+}\right)$if and only if

$$
F(\lambda)=\int_{0}^{\infty} f(x) e^{i x \lambda} d x
$$

with some $f \in L^{2}(0, \infty)$. In $[4$ the following sampling formula for a Hardy function was established.

Theorem 3. Let $F \in H^{2}\left(\mathbb{C}^{+}\right)$. Then for any $\lambda \in \mathbb{C}^{+}$,

$$
F(\lambda)=\sum_{n \geq 0} c_{n}(-i \lambda) \sum_{k=0}^{n} a_{n k} F\left(\lambda_{k}\right),
$$

where

$$
\begin{aligned}
\lambda_{k} & =i\left(k+\frac{1}{2}\right) \\
c_{n}(-i \lambda) & =(2 n+1) \frac{\left(\frac{1}{2}+i \lambda\right)_{n}}{\left(\frac{1}{2}-i \lambda\right)_{n+1}} \\
a_{n k} & =\frac{(-n)_{k}(n+1)_{k}}{(k !)^{2}} \\
(x)_{n} & =\frac{\Gamma(x+n)}{\Gamma(x)}=x(x+1) \cdots(x+n-1) .
\end{aligned}
$$


In this paper we need a similar sampling formula, but for another class of functions.

Theorem 4. Let

$$
F(s)=\int_{0}^{\infty} e^{-s t} f(t) d t
$$

be the Laplace transform of a function $f$ such that $e^{-\epsilon t}|f(t)| \in L^{1}(0, \infty)$ for any $\epsilon>0$. Then

$$
F(s)=\sum_{n=0}^{\infty} c_{n}\left(s+\frac{1}{2}-\epsilon\right) \sum_{k=0}^{n} a_{n k} F(k+\epsilon)
$$

where

$$
\begin{aligned}
a_{n k} & =\frac{(-n)_{k}(n+1)_{k}}{(k !)^{2}}, \\
c_{n}(x) & =\frac{(2 n+1)(1 / 2-x)_{n}}{(1 / 2+x)_{n+1}} .
\end{aligned}
$$

The series in (10) converges uniformly on any compact subset in the half plane $\operatorname{Re} s>\frac{1}{2}+\epsilon$ and moreover

$$
F(s)-\sum_{n=0}^{N} c_{n}\left(s-\epsilon+\frac{1}{2}\right) \sum_{k=0}^{n} a_{n k} F(k+\epsilon)=O\left(N^{-\left(\operatorname{Re} s-\epsilon-\frac{1}{2}\right)}\right), \quad N \rightarrow \infty .
$$

Proof. Setting $e^{-t}=x$ we have

$$
\begin{aligned}
F(s) & =\int_{0}^{\infty} e^{-s t} f(t) d t \\
& =\int_{0}^{1} g(x) x^{s-1 / 2} d x, \quad \operatorname{Re} s>0,
\end{aligned}
$$

where $g(x):=f(-\ln x) x^{-1 / 2}$. Since $e^{-\epsilon t} f(t) \in L^{1}(0, \infty)$ if and only if $x^{\epsilon-1 / 2} g(x) \in$ $L^{1}(0,1)$, both integrals in (13) are absolutely convergent.

Now expand $x^{s-\epsilon}$ in normalized Legendre polynomials $\sqrt{2 n+1} P_{n}(1-2 x), \quad 0 \leq$ $x \leq 1$. To this end, first expand the principal branch of the power function $\left(\frac{1-y}{2}\right)^{a-2},-1<y<1, \operatorname{Re} a>3 / 2$, in Legendre polynomials $P_{n}(y)$ :

$$
\left(\frac{1-y}{2}\right)^{a-2}=\sum_{n=0}^{\infty} \frac{2 n+1}{2} \alpha_{n} P_{n}(y)
$$

where [6, formula 2.17.1.10]

$$
\alpha_{n}=\int_{-1}^{1}\left(\frac{1-y}{2}\right)^{a-2} P_{n}(y) d y=\frac{2(2-a)_{n}}{(a-1)_{n+1}} .
$$


Taking $y=1-2 x$ and $a=s+2-\epsilon$ in (14), where $\epsilon$ is the same as in (13), yields (for $0<x<1$ and $\operatorname{Re} s>-\frac{1}{2}+\epsilon$ )

$$
x^{s-\epsilon}=\sum_{n=0}^{\infty} \frac{(2 n+1)(\epsilon-s)_{n}}{(s+1-\epsilon)_{n+1}} P_{n}(1-2 x)=\sum_{n=0}^{\infty} c_{n}\left(s+\frac{1}{2}-\epsilon\right) P_{n}(1-2 x),
$$

where $c_{n}$ is defined by (11). Plugging the well-known explicit representation

$$
P_{n}(1-2 x)=\sum_{k=0}^{n} a_{n k} x^{k}, \quad a_{n k}=\frac{(-n)_{k}(n+1)_{k}}{(k !)^{2}}
$$

into (15) yields

$$
x^{s-\epsilon}=\sum_{n=0}^{\infty} c_{n}\left(s+\frac{1}{2}-\epsilon\right) \sum_{k=0}^{n} a_{n k} x^{k}, \quad 0<x<1, \quad \operatorname{Re}(s)>-\frac{1}{2}+\epsilon .
$$

If, in addition, $\operatorname{Re} s>\epsilon+\frac{1}{2}$, then $x^{s-\epsilon}$ satisfies a Lipschitz condition of order $>1 / 2$, and therefore the series (16) converges uniformly on $[0,1]$ (see [9]). Hence, if $g$ is any function from $L^{1}\left([0,1], x^{\epsilon-1 / 2} d x\right)$ and $\operatorname{Re} s>\frac{1}{2}+\epsilon$, then

$$
\begin{aligned}
\int_{0}^{1} g(x) x^{s-1 / 2} d x & =\int_{0}^{1} g(x) x^{\epsilon-1 / 2} x^{s-\epsilon} d x \\
& =\int_{0}^{1} g(x) x^{\epsilon-1 / 2}\left(\sum_{n=0}^{\infty} c_{n}(s+1 / 2-\epsilon) \sum_{k=0}^{n} a_{n k} x^{k}\right) d x
\end{aligned}
$$

where the series converges uniformly on $[0,1]$. Thus, we can interchange the order of integration and infinite summation in (17) to get

$$
\begin{aligned}
F(s) & =\sum_{n=0}^{\infty} c_{n}(s+1 / 2-\epsilon)\left(\int_{0}^{1} g(x) \sum_{k=0}^{n} a_{n k} x^{k-\frac{1}{2}+\epsilon}\right) d x \\
& =\sum_{n=0}^{\infty} c_{n}(s+1 / 2-\epsilon) \sum_{k=0}^{n} a_{n k} \int_{0}^{1} g(x) x^{k-\frac{1}{2}+\epsilon} d x \\
& =\sum_{n=0}^{\infty} c_{n}(s+1 / 2-\epsilon) \sum_{k=0}^{n} a_{n k} F(k+\epsilon) .
\end{aligned}
$$

Thus we can interpolate the function $F$ by the sequence of its values $\{F(k+\epsilon), k=0,1, \ldots\}$, the series (18) being uniformly convergent on any compact subset in the half-plane $\operatorname{Re} s>\frac{1}{2}+\epsilon$.

The function $x^{s-\epsilon}$ is differentiable $[\operatorname{Re} s-\epsilon]$ times $([x]$ is the integer part of $x)$, and its $[\operatorname{Re} s-\epsilon]$ th-order derivative satisfies the Lipschitz condition of order $\{\operatorname{Re} s-\epsilon\}$, where $\{x\}$ is the mantissa of $x$. Then by Theorem 4.10 in [9] we arrive at

$$
F(s)-\sum_{n=0}^{N} c_{n}\left(s+\frac{1}{2}-\epsilon\right) \sum_{k=0}^{n} a_{n k} F(k+\epsilon)=O\left(\frac{1}{N^{\operatorname{Re} s-\epsilon-\frac{1}{2}}}\right), \quad \operatorname{Re} s>\frac{1}{2}+\epsilon .
$$




\section{The MAIN Result}

The following interpolation theorem is our main result, which for the reader's convenience we state in a self-contained form.

Theorem 5. Let $q(x), x \geq 0$, be a real potential in $l^{\infty}\left(L^{1}\right)$, i.e.,

$$
\|q\|:=\sup _{x \geq 0} \int_{x}^{x+1}|q(t)| d t<\infty
$$

and let $m(\lambda)$ be the Dirichlet Titchmarsh-Weyl m-function associated with the Schrödinger operator $-d^{2} / d x^{2}+q(x)$ on $L^{2}(0, \infty)$. Ther 1

$$
\begin{aligned}
& m(\lambda)-i \sqrt{\lambda} \\
& =\sum_{n=0}^{\infty} c_{n}\left(-i \sqrt{\lambda}-\beta_{0}+1\right) \sum_{k=0}^{n} a_{n k}\left(m\left(-\omega_{k}^{2}\right)+\omega_{k}\right),
\end{aligned}
$$

for any $\lambda$ from the parabolic domain $\left\{\lambda \in \mathbb{C}: \operatorname{Im}^{2} \lambda>4 \beta_{0}^{2} \operatorname{Re} \lambda+4 \beta_{0}^{4}\right\}$, where

$$
\begin{aligned}
\beta_{0} & :=\max \{\sqrt{2\|q\|}, e\|q\|\}+\frac{1}{2}+\epsilon, \epsilon>0, \\
c_{n}(x) & =(2 n+1) \frac{\left(\frac{1}{2}-x\right)_{n}}{\left(\frac{1}{2}+x\right)_{n+1}},(x)_{n}=x(x+1) \cdots(x+n-1), \\
a_{n k} & =\frac{(-n)_{k}(n+1)_{k}}{(k !)^{2}}, \\
\omega_{k} & =k+\beta_{0}-\frac{1}{2} .
\end{aligned}
$$

Proof. By equation (4.5) of [2,

$$
\begin{aligned}
A(x) & =q(x)+a(x) \\
|a(x)| & \leq \frac{1}{2}\left(\int_{0}^{x}|q(t)| d t\right)^{2}\left\{\exp (2 \sqrt{2\|q\|} x)+\frac{1}{\sqrt{2 \pi}} \exp (2 e\|q\| x)\right\} \\
& \leq \frac{1}{2}\left(\int_{0}^{x}|q(t)| d t\right)^{2}\left(1+\frac{1}{\sqrt{2 \pi}}\right) e^{2 \gamma x}, \quad \gamma:=\max \{\sqrt{2\|q\|}, e\|q\|\} .
\end{aligned}
$$

If $n$ is an integer such that $x \leq n<x+1$, and $\sup _{x>0} \int_{x}^{x+1}|q(t)| d t=\|q\|<\infty$, then

$$
\int_{0}^{x}|q(t)| d t \leq \int_{0}^{n}|q(t)| d t=\sum_{k=0}^{n-1} \int_{k}^{k+1}|q(t)| d t \leq\|q\| n<\|q\|(x+1) .
$$

\footnotetext{
${ }^{1}$ The branch cut in $\sqrt{\lambda}$ is performed along $(0, \infty)$.
} 
Hence, $e^{-2 \beta x} a(x) \in L^{1}(0, \infty)$ for any $\beta>\gamma$. On the other hand, for any $\beta>0$ and $q \in l^{\infty}\left(L^{1}\right)$ we have

$$
\begin{aligned}
\int_{0}^{\infty} e^{-\beta t}|q(t)| d t & =\frac{\beta}{e^{\beta}-1} \int_{0}^{\infty}|q(t)| \int_{t-1}^{t} e^{-\beta x} d x d t \\
= & \frac{\beta}{e^{\beta}-1} \int_{0}^{\infty} e^{-\beta x} \int_{x}^{x+1}|q(t)| d t d x \\
& +\frac{\beta}{e^{\beta}-1} \int_{-1}^{0} e^{-\beta x} \int_{0}^{x+1}|q(t)| d t d x \\
\leq & \frac{\|q\|}{\beta}+\|q\|
\end{aligned}
$$

Consequently,

$$
\int_{0}^{\infty} e^{-\epsilon x}\left|A\left(\frac{x}{2}\right)\right| e^{-\gamma x} d x<\infty
$$

for any $\epsilon>0$. One has

$$
m\left(-\kappa^{2}\right)+\kappa=-\frac{1}{2} \int_{0}^{\infty} A\left(\frac{x}{2}\right) e^{-\kappa x} d x=-\frac{1}{2} \int_{0}^{\infty} A\left(\frac{x}{2}\right) e^{-\gamma x} e^{-(\kappa-\gamma) x} d x
$$

If we set $s=\kappa-\gamma$, equation (20) becomes

$$
m\left(-(s+\gamma)^{2}\right)+(s+\gamma)=-\frac{1}{2} \int_{0}^{\infty} A\left(\frac{x}{2}\right) e^{-\gamma x} e^{-s x} d x
$$

where the last integral converges for any $s$ with Re $s>0$. Applying Theorem 4 to $F(s)=m\left(-(s+\gamma)^{2}\right)+(s+\gamma)$ yields

(22) $m\left(-(s+\gamma)^{2}\right)+(s+\gamma)$

$$
=\sum_{n=0}^{\infty} c_{n}(s+1 / 2-\epsilon) \sum_{k=0}^{n} a_{n k}\left[m\left(-\left(k+\beta_{0}-\frac{1}{2}\right)^{2}\right)+k+\beta_{0}-\frac{1}{2}\right] \text {, }
$$

for $\operatorname{Re} s>\frac{1}{2}+\epsilon$. Substituting $s+\gamma=-i \sqrt{\lambda}$ in (22), we arrive at (19). It remains to deal with the domain of convergence of (19).

For our new spectral variable $\lambda$, if $\lambda$ is in the parabolic domain $\operatorname{Im}^{2} \lambda>4 \beta_{0}^{2} \operatorname{Re} \lambda+$ $4 \beta_{0}^{4}$, then

$$
\begin{aligned}
|\lambda|^{2} & >\left(\operatorname{Re} \lambda+2 \beta_{0}^{2}\right)^{2} \\
& =\left(|\lambda| \cos \theta+2 \beta_{0}^{2}\right)^{2},
\end{aligned}
$$

where $\theta=\arg \lambda \in[0,2 \pi)$. Thus

$$
|\lambda|(1-\cos \theta)>2 \beta_{0}^{2}
$$

and, consequently,

Therefore,

$$
\sqrt{|\lambda|} \sin \frac{\theta}{2}>\beta_{0}
$$

$$
\operatorname{Im} \sqrt{\lambda}>\beta_{0}
$$

Recalling that $\beta_{0}=\gamma+\frac{1}{2}+\epsilon$, we see that the latter inequality becomes

$$
\operatorname{Re} s=\operatorname{Re}(-i \sqrt{\lambda})-\gamma>\frac{1}{2}+\epsilon \text {. }
$$


Thus if $\lambda$ is in the parabolic domain, then $\operatorname{Re} s>\frac{1}{2}+\epsilon$, which is exactly the domain of convergence of (22). Theorem 5 is proved.

Remark 6. Theorems 1 and 5 readily yield an algorithm for producing a rational approximation to the Titchmarsh-Weyl $m$-function. Indeed, given a potential $q(x)$, by solving the integral equation (8) one evaluates $A(x, y)$, whose diagonal values give the $A$-amplitude by (7). For a sampling set of equally spaced real points $\omega_{k}$ from Theorem 5 , one then evaluates $m\left(-\omega_{k}^{2}\right)+\omega_{k}$ by (6). Finally, the TitchmarshWeyl $m$-function $m(\lambda)$ can be computed from (19) for any $\lambda$ from the domain $\operatorname{Im}^{2} \lambda>4 \beta_{0}^{2} \operatorname{Re} \lambda+4 \beta_{0}^{4}$. Since the integral in (6) and the series in (19) are both absolutely convergent and the functions $c_{n}\left(-i \sqrt{\lambda}-\beta_{0}+1\right)$ are all rational with respect to $\sqrt{\lambda}$, we arrive at a rational approximation of $m(\lambda)$. However, such rational approximations are not Herglotz functions, in contrast to the TitchmarshWeyl $m$-function itself.

Remark 7. The sequence $\left\{\omega_{k}\right\}$ in our interpolation formula (19) is not what is commonly referred to as an interpolating sequence (see, e.g., 1]). An interpolating sequence typically consists of an arbitrary set of points in the upper half plane or the unit disk which are used to interpolate analytic functions from Hardy spaces (the Nevanlinna-Pick problem). An important ingredient of the Nevanlinna-Pick theory is the Blaschke product with zeros made up of the interpolating sequence. Our sequence $\left\{\omega_{k}\right\}$ lies on the negative part of the real line and hence does not satisfy the Blaschke condition. Switching from the variable $\lambda$ to $\sqrt{\lambda}$ does not help, as the sequence $\left\{i \sqrt{-\omega_{k}}\right\}$ is not subject to the Blaschke condition either. Moreover the Titchmarsh-Weyl $m$-function $m(\lambda)$ need not be in a Hardy space $H^{p}\left(\mathbb{C}^{+}\right)$for any $p$ with $0<p \leq \infty$. Indeed, it follows from its Herglotz representation

$$
m(\lambda)=\operatorname{Re} m(i)+\int_{\mathbb{R}}\left(\frac{1}{t-\lambda}-\frac{t}{1+t^{2}}\right) d \mu,
$$

where $\mu$ is a positive measure subject to

$$
\int_{\mathbb{R}} \frac{d \mu}{1+t^{2}}<\infty
$$

that a pure point of $\mu$ produces a term in (23) which is not in $H^{p}\left(\mathbb{C}^{+}\right)$.

\section{ACKNOWLEDGEMENT}

The authors deeply thank the referee for numerous valuable comments that led to improvement of the paper's presentation.

\section{REFERENCES}

[1] J. Agler and J. E. McCarthy. Pick Interpolation and Hilbert Function Spaces. Graduate Studies in Mathematics, 44. American Mathematical Society, Providence, RI, 2002. MR 1882259 (2003b:47001)

[2] S. Avdonin, V. Mikhaylov, and A. Rybkin. The boundary control approach to the TitchmarshWeyl $m$-function. I. The response operator and the $A$-amplitude. Comm. Math. Phys. 275 (2007), no. 3, pp. 791-803. MR.2336364 (2008g:93083)

[3] A. Boumenir and Vu Kim Tuan. The interpolation of the Titchmarsh-Weyl function. J. Math. Anal. Appl. 335 (2007), pp. 72-78. MR2340306 (2008f:34059)

[4] A. Boumenir and Vu Kim Tuan. Sampling eigenvalues in Hardy spaces. SIAM J. Numer. Anal. 45 (2007), no. 2, pp. 473-483. MR2300282 (2008c:65180) 
[5] F. Gesztesy and B. Simon. A new approach to inverse spectral theory, II. General real potentials and the connection to the spectral measure. Ann. of Math. (2) 152 (2000), no. 2, pp. 593-643. MR.1804532 (2001m:34185b)

[6] A. P. Prudnikov, Yu. A. Brychkov, and O. I. Marichev. Integrals and Series. Special Functions (Russian). Nauka, Moscow, 1983, English translation: Integrals and series. Vol. 3. More special functions. Gordon and Breach Science Publishers, New York, 1990. MR 1054647 (91c:33001)

[7] A. G. Ramm. Recovery of the potential from I-function. C. R. Math. Rep. Acad. Sci. Canada 9 (1987), no. 4, pp. 177-182. MR896977 (88k:34012)

[8] B. Simon. A new approach to inverse spectral theory, I. Fundamental formalism. Ann. of Math. (2) 150 (1999), no. 3, pp. 1029-1057. MR1740987 (2001m:34185a)

[9] P. K. Suetin. Classical Orthogonal Polynomials (Russian), Second edition. Nauka, Moscow, 1979. MR548727 (80h:33001)

Department of Mathematics and Statistics, University of Alaska Fairbanks, P.O. Box 756660, Fairbanks, Alaska 99775

E-mail address: ffavr@uaf.edu

Department of Mathematics, University of West Georgia, Carrollton, Georgia 30118

E-mail address: vu@westga.edu 